\title{
Vingt ans de créations nues au Québec
}

\section{Daniel Léveillé et Claire Vionnet}

\section{(2) OpenEdition \\ Journals}

\section{Édition électronique}

URL : http://journals.openedition.org/danse/3813

DOI : $10.4000 /$ danse.3813

ISSN : 2275-2293

Éditeur

ACD - Association des Chercheurs en Danse

\section{Référence électronique}

Daniel Léveillé et Claire Vionnet, « Vingt ans de créations nues au Québec », Recherches en danse [En ligne], Entretiens, mis en ligne le 29 avril 2021, consulté le 01 mai 2021. URL : http:// journals.openedition.org/danse/3813; DOI : https://doi.org/10.4000/danse.3813

Ce document a été généré automatiquement le 1 mai 2021.

association des Chercheurs en Danse 


\title{
Vingt ans de créations nues au Québec
}

\author{
Daniel Léveillé et Claire Vionnet
}

1 J'ai rencontré Daniel en juillet 2019 à Montréal, lorsque je menais une ethnographie sur l'intimité en danse contemporaine. L'association ordinaire entre intimité et nudité a conduit mes interlocuteurs·trices de terrain à énumérer les chorégraphes montréalais qui travaillaient avec le nu, tels que Dave St Pierre ou Benjamin Kamino.

Daniel Léveillé m'a été présenté comme le chorégraphe québécois de la nudité. Pionnier au Québec, le nu est au cœur de ses créations depuis 2001. Formé par la compagnie Groupe Nouvelle Aire de Montréal, Daniel travaille comme chorégraphe indépendant depuis 1974, puis fonde Daniel Léveillé Danse en 1991. Parallèlement à sa compagnie, il enseigne de 1988 à 2012 la création et l'interprétation à l'Université du Québec de Montréal.

2 J'ai interrogé Daniel sur sa découverte de la nudité, comment elle affecte le processus de création, ses significations, son association aux notions d'intimité et de sexualité, et les politiques de réception. L'entretien a mis en exergue de quelle manière le nu ne signifie que dans son enchevêtrement avec d'autres médias, tels que la lumière, le geste et l'espace.

Cet entretien a été retranscrit puis réorganisé et retravaillé en collaboration avec le chorégraphe.

\section{La nudité, un choix chorégraphique}

CLAIRE VIONNET : Daniel, la nudité est au cœur de ton travail chorégraphique. Qu'est-ce qui t'a amené à travailler avec le nu?

Daniel Léveillé : En mai 1981, j'ai créé une pièce théâtrale intitulée L'Étreinte, basée sur un texte de l'écrivain français Yves Navarre. Il y avait un long pas de deux entre deux garçons nus, image miroir de ce que racontait le texte de Navarre. J'avais utilisé la lumière de manière tamisée. Elle provenait surtout de l'arrière de la scène, donc on ne voyait pas vraiment les sexes. 
En février 2001, j'ai créé Amour, acide et noix. Quand j'ai créé cette pièce-là, je n'avais pas l'idée d'utiliser le corps nu. Ce que je savais, c'est que je voulais traiter du corps et donc laisser voir le plus possible de chair. Puis à une étape des répétitions, j'ai mis des gants blancs et demandé aux danseurs de faire un enchaînement nu, juste par curiosité, pour voir ce que ça donnait. Ça a été un choc pour nous tous, parce que ce n'était plus du tout la même pièce. Ça a été vraiment une révélation. Avec les sousvêtements, la pièce était très sexy, les danseurs étant jeunes et plutôt beaux. Avec la nudité s'est révélée une sorte de fragilité et par là, les possibilités de lecture de l'œuvre s'en sont trouvées démultipliées. La lumière était très blanche et crue, laissant voir les corps sous toutes les coutures, sans fausse pudeur. Elle servait plutôt à sculpter les corps qu'à créer une ambiance.

C'est cette pièce qui m'a fait connaître partout. C'est par elle que s'est d'abord développée ma carrière internationale. On l'a tournée des centaines de fois, au point que certains chorégraphes européens ont eux aussi, à un moment donné, voulu utiliser la nudité : des mouvements simples, aucune ambiguïté ou malaise sexuel, que des corps nus dans un espace nu, lui aussi, mais très travaillé.

CLAIRE VIONNET: Tu as donc pris conscience du sens particulier que le nu donnait à la chorégraphie. La même gestuelle peut signifier autre chose si elle est nue ou si elle est habillée. Tu évoques aussi la lumière comme médium important pour transformer le sens : tamisée, elle cache les sexes; blanche, elle les expose sans pudeur. Tu dis que la lumière a cette capacité de sculpter le corps. C'est donc un peu par hasard que tu découvres la nudité lors d'un processus de création en 1981. Tu y reviens vingt ans plus tard de manière intensive, de sorte qu'exceptées deux pièces, toutes tes créations depuis 2001 mettent en scène des nus intégraux.

Daniel Léveillé : Oui, jusqu'en 2012, la nudité a été le seul costume de mes pièces, je ne me posais plus la question des costumes. En 2012, j'ai créé Solitudes solo. Je pensais que cette pièce serait nue comme les précédentes, pour finalement constater que ce n'était pas pertinent, que ça n'ajoutait rien de plus. Cette pièce a été dansée avec des sous-vêtements. C'était intéressant, cela permettait de travailler la couleur. Pièce séquentielle, comme une série de dessins. Un solo, puis un autre, etc. Pour chacun des solos, en changeant la couleur des vêtements, on avait presque l'impression que c'était un autre danseur. Puis en 2015, j'ai créé Solitudes duo dans la même veine, une suite séquentielle de duos. Pour ces deux pièces, je n'ai pas utilisé la nudité parce que je trouvais que ça venait brouiller la lecture. Récemment, j'ai créé Quatuor tristesse (2018) et je suis revenu à la nudité. Donc oui, pour moi, la question de la nudité se pose pour chaque création.

CLAIRE VIONNET: La nudité n'est donc pas un hasard mais répond à un choix chorégraphique...

Daniel Léveillé : Aujourd'hui, alors que presque toutes les barrières et limites ont été dépassées - parfois à la limite du tolérable - la nudité devient un outil comme un autre, assez banal. L'enjeu, pour un chorégraphe ou un metteur en scène qui utilise la nudité, relève plutôt de la pertinence de son utilisation, sans donner l'impression que le geste est gratuit. Le chorégraphe a ses raisons de l'utiliser, comme d'utiliser des souliers à talons hauts, des vêtements victoriens, des béquilles ou des prothèses. Ça devient un outil comme un autre et qui est perçu, j'ose croire, de la même manière. La nudité, pour certains encore, ça trouble un peu, mais de moins en moins je crois, on s'y habitue. 
CLAIRE Vionnet : Tu parles de la nudité comme outil et médium. Pourtant, il faut la justifier et expliquer en quoi elle est pertinente. Même si elle n'a plus la fonction de dépasser les limites, devient-elle pour autant banale? Tu sembles dire qu'elle est justement choisie pour raconter une histoire spécifique. $Y$ a-t-il une différence entre une gestuelle habillée et une gestuelle nue?

Daniel Léveillé : Aucune. Pour moi, aucune. Pour la première pièce de nus que j'ai créée, je n'avais pas en tête la nudité, donc je ne me suis pas posé la question. Et je ne me pose jamais la question. Je peux envoyer les corps dans toutes les directions. Beaucoup de danseurs aiment particulièrement la nudité dans les pas de deux ou dans les portés. Peut-être moins s'il y a beaucoup de sueur, car ça peut devenir difficile - mais même avec la sueur ça reste quand même mieux qu'avec le vêtement, car le vêtement glisse toujours. Le vêtement glisse sous la main. C'est difficile d'agripper le danseur. Il est plus facile de voir où poser les mains pour un porté par exemple. On n'est pas troublé par la hauteur de la ceinture à la taille. On voit le corps et on voit précisément où doit s'exécuter la prise.

Une fois, j'ai failli enlever deux mouvements. Quand j'ai vu un enchaînement nu, j'ai pensé « oh là là, un tour attitude par des garçons avec le pénis qui part en zigzag ». Puis un autre mouvement, où les danseurs étaient pliés à la seconde, dos au public, avec le cul vraiment sorti. Mais finalement, je me suis dit « je ne serai pas le premier censeur » et j'ai décidé de laisser ces mouvements. Plusieurs sont venus me parler de ces moments, me disant que c'étaient des moments très forts. Et il y a la danse des orifices... Il y a un petit malaise au début, disons pour les cinq premières minutes. Certains spectateurs n'osent pas regarder au début ou alors d'autres ne regardent que les sexes. Puis quelque chose se débloque et tous en viennent immanquablement à oublier la nudité. Tous les spectateurs arrivent à oublier, après un certain temps, que les danseurs sont nus.

Pour moi, il n'y a aucune différence entre créer une gestuelle pour des danseurs nus ou habillés. Une fois que le pire a été traversé, les moments les plus délicats passés (échappées, arabesques, tours attitudes, grands écarts, jambes à la seconde) - autant pour les garçons que les filles - à ce moment-là, il n'y a plus aucune gêne ou malaise chez les danseurs.

\section{Nudité versus vêtement : neutralité et culturalisme}

\section{CLAIRE VIONNET : Qu'est-ce que le nu te permet de dire de plus que le corps habillé ?}

Daniel Léveillé : La nudité n'est pas neutre. Le vêtement non plus d'ailleurs. Il est impossible d'habiller les danseurs sans, d'une manière ou d'une autre, les inscrire quelque part. C'est ça qui m'énervait avec le vêtement. Ce que j'aime bien de la nudité, c'est qu'on est au cœur du sujet de l'être humain, on parle du corps esthétique et donc d'une certaine beauté, sinon d'une certaine vérité. Dans mon travail, on peut voir la jeunesse des corps, le corps vertueux, le corps parfait. Beaucoup de chorégraphes utilisent certains vêtements en pensant qu'on n'y fera pas attention et qu'ils sont neutres. Mais ils ne sont jamais neutres. Même un t-shirt n'est pas neutre. Il n'y a rien qui soit neutre. Avec le vêtement, on inscrit les danseurs à la fois dans le temps, mais surtout socialement. Avec la nudité, on évite ce piège. On est moins dans un groupe d'individus qui appartiendraient à une tribu, on est juste dans l'être humain. 
CLAIRE VIONNET: Donc tu penses que le nu permet de parler de l'humain au-delà des cultures car il n'y a plus d'habit pour renvoyer à une communauté particulière. Pour toi, le corps nu libère du poids culturel. L'anthropologue Terence Turner écrit que l'être humain naît nu, mais vit habillé, car le vêtement fait de l'humain un homme de sens. Turner dit que le vêtement est un medium culturel qui attribue une identité sociale. La surface du corps est la surface symbolique sur laquelle se joue le récit de la socialisation?

Daniel Léveillé : D’ailleurs il n'y a pas la nudité en danse, mais des nudités. Selon la proposition ou l'écriture chorégraphique, le sens peut en être très différent. Et la raison de l'utilisation de la nudité par le chorégraphe ou les danseurs peut être très différente aussi pour les uns ou les autres. On peut même vouloir l'utiliser pour choquer. Je n'ai pas pu voir d'autres spectacles à la biennale de Venise (2019) parce que je n'ai pas été là assez longtemps mais en feuilletant la brochure, j'ai noté un intérêt porté sur les corps atypiques. Pour une création, il y avait une photo de nus : un homme immensément grand et une toute petite femme naine, belle, mais très petite. C'était quand même wow! Il y avait une autre pièce, c'était un cul-de-jatte qui était aussi nu par moment. Donc on voit la volonté de montrer des corps atypiques, et de les montrer nus aussi. Ce n'est pas du tout ma tasse de thé. Ce n'est pas mon propos de chorégraphe, ni mon vocabulaire, mais je serais curieux comme spectateur d'aller voir ces pièces-là.

CLAiRE vionnet : Tu as évoqué la polysémie du nu. Roland Huesca dit que le nu des années 1960 aux États-Unis était rebelle et transgressif. II perd sa dimension politique dans les années 1990 en France. Les chorégraphes s'intéressent alors aux composantes du spectacle (lumière, costume) pour déjouer les évidences. C'est pour interroger la matérialité du corps qu'ils l'ont déshabillé, mettant en scène les corps sous toutes leurs formes, pas seulement les corps esthétiques des danseurs².

Daniel Léveillé : Je suis très sensible à la beauté, quelque définition qu'on puisse lui donner. La plupart de mes danseurs - sinon tous - se doivent d'être infiniment beaux. La beauté, c'est d'abord l'enveloppe mais c'est plus que ça. C'est une lumière qui émane d'une personne. Mais s'il n'y a pas l'enveloppe, il n'y a pas la beauté.

Pourtant les danseurs eux-mêmes, que ce soient les garçons ou les filles, même s'ils sont beaux, eux ne se trouvent jamais très beaux. L'une des raisons pour laquelle on s'habille, n'est-ce pas pour cacher ses défauts? «J'ai le cul trop gros, j'ai les bras trop longs, j'ai pas de seins", peu importe. Et on choisit le vêtement qui atténue ces défauts. Accepter de s'exposer nu, c'est accepter entièrement le corps qu'on a. C'est une très belle leçon d'interprétation. Tous les danseurs devraient toucher à ça, au moins une fois au cours de leur vie professionnelle.

Mon travail avec le nu rejoint la sculpture antique. Un Apollon, une Vénus, c'est le corps et la beauté du corps. La nudité permet aussi de souligner la complexité du corps. Le spectateur est amené à porter plus d'attention aux différentes composantes du corps humain. Ce n'est pas un bloc de marbre qu'il voit. Quand on voit des corps nus, on devient plus sensibles à la variété et à la complexité des diverses composantes du corps humain. J'ai la prétention d'exposer le plus possible d'un danseur sur scène : son intelligence, sa sensibilité, ses émotions, sa force, ses doutes et ceci sans aucun filtre. Le moins on utilise de filtres, le mieux c'est.

\section{Revêtir le costume du nu}


CLAiRe vionnet : Donc le dénudement physique permet un dépouillement émotionnel... Pourtant, beaucoup de danseurs me disent que danser nu, c'est comme danser avec un costume de nu. Est-ce une manière de protéger son intimité ?

Daniel Léveillé : Les danseurs savent que la nudité glorifie les corps, mais aussi que ça les fragilise. Ils savent également qu'elle a une incidence qui n'est pas neutre. Donc, c'est comme revêtir un costume. Ce qui est bien, c'est le fait d'en parler de cette manière-là, ça les protège. Je vais dire par exemple : «on fait un enchaînement en costumes » et le danseur traduit par : «je revêts mon armure », plutôt que de se dire «je me fous à poil».

Il y a une telle proximité entre les corps des danseurs que cela ne les défie plus vraiment en termes d'intimité. C'est ailleurs que l'intimité se vit, par exemple lors de tournées où l'on vit les uns avec les autres pendant des semaines. Tu quittes ton partenaire amoureux, tu vis dans une sorte de bulle avec les autres danseurs. On mange tous ensemble le soir après les spectacles, il y a une intimité au sein d'une compagnie de danse, c'est comme une autre vie, une vie parallèle. Il y a aussi beaucoup de sensibilité liée aux représentations des spectacles: tu danses, c'est challengeant et c'est difficile; il y a les bons soirs et les moins bons spectacles; parfois le travail est moins bien reçu; il y a parfois de mauvaises critiques. Tout ça te challenge. C'est dans tout ça que l'intimité ressort.

CLAIRE VIONNET : Donc chez les danseurs professionnels, la nudité relève moins de l'intime que la proximité des liens au sein de la compagnie. Et les danseurs associent le nu à un vêtement. J'imagine qu'il y a un apprentissage pour apprivoiser ce sens de la nudité. Comment tes danseurs vivent-ils leur nudité sur scène?

Daniel Léveillé : Ça prend un certain temps pour s'habituer. Il faut se demander : «pourquoi veux-tu aller sur scène? Qu'est-ce que tu veux faire avec ça? Qu'est-ce que tu veux montrer? » Si tu choisis de faire ça de ta vie, il faut accepter que d'abord, c'est ton corps que tu dois montrer et ce, sans fausse pudeur. Il ne faut pas tenter de cacher quoi que ce soit.

Ça peut être très différent d'un danseur à l'autre. Certains danseurs sont excessivement à l'aise avec la nudité et d'autres moins. Ceux qui ne l'ont jamais fait vont se sentir un peu plus timides. Ce que je vais faire pour m'assurer que, quand viendra le moment de danser devant une salle entière, le danseur ne va pas être en retrait ou nerveux, je vais lui demander quelle est la pire personne devant laquelle il pourrait s'imaginer danser nu. En général c'est le père pour les filles, et la mère pour les garçons, ou alors leur copine. Je propose de demander à ces gens-là de venir assister à une répétition pour faire en sorte que cette pression soit libérée si les proches sont dans la salle lors de la première. Après ce passage devant les parents, ça devient presque banal de danser devant quatre cents personnes anonymes.

CLAIRE VIONNET : Ce tabou de la nudité intra-familial renvoie-t-il aux théories de l'inceste et du tabou sexuel au sein de la famille pour favoriser l'exogamie?

Daniel Léveillé : C'est surtout lié au fait qu'il y ait eu ou non de la nudité dans la famille. Chez un danseur, tout le monde se promenait partout nu chez lui. Pour lui ça a été absolument banal. Donc aucun problème de se promener nu devant ses parents, sauf peut-être pour la courte période de l'adolescence. Quand les enfants ont vu leurs parents nus, tout naturellement, ça démythifie beaucoup. Dans d'autres familles, on ne sort jamais nu de sa chambre à coucher. 
Ce qui est le plus difficile à faire passer sur scène, ce n'est pas la nudité, mais le fait de se dévêtir. Ou se rhabiller. C'est peut-être là où on frôle le plus la sexualité, ou la sexualisation des corps. D'ailleurs, quand ils viennent saluer, mes danseurs sont habillés. La toute première fois, j'avais oublié de leur transmettre cette consigne et ils sont venus saluer nus, cela s'avérait plus rapide. Ce fut la catastrophe! Il est absolument nécessaire de faire une coupure. Il faut leur laisser le temps de se rhabiller. La nudité, c'est le costume de la pièce mais aux saluts, on retrouve les danseurs.

On ne répète presque jamais nus. Il n'y a pas vraiment de raison de le faire, sauf quand on doit fixer la lumière, ou pour certains portés. Ou alors pour un nouveau danseur, pour que ce ne soit pas trop un choc durant le spectacle, pour qu'il s'habitue à cette proximité-là avec ses partenaires, on va faire deux ou trois enchaînements nus. Parfois les sexes passent très près du visage et il y a la sueur et les odeurs aussi. Sinon, il n'y a pas de raison de répéter nu.

CLAiRe vionnet: Ce que tu évoques rejoint la distinction de l'historien de l'art Clark Kenneth entre le nu quotidien (naked) et le nu artistique (nude). L'anglais opère d'ailleurs une distinction terminologique que ne fait pas le français. Kenneth écrit que le corps nu de la vie ordinaire est embarrassant. Par contre, transformé par l'art, le nu artistique n'est lié ni à la gêne, ni à l'inconfort ${ }^{3}$.

\section{Nudité et sexualité}

CLAIRE VIONNet : Perçois-tu une différence genrée dans l'expérience de la nudité ?

Daniel Léveillé : Oui, on ne parle pas de la même nudité pour un homme ou pour une femme. Pour le danseur ou la danseuse pourtant, c'est exactement la même chose, mais pas toujours dans le regard de celle ou de celui qui regarde. J'ai toujours été fasciné de voir qu'en général, une femme nue se sente plus nue qu'un homme nu. Pourtant l'homme en révèle bien plus que la femme! Droit debout, le sexe de la femme reste caché, alors que celui de l'homme est exposé. Je crois que c'est d'abord lié au regard des hommes sur le corps des femmes ou au regard des femmes sur le corps des femmes elles-mêmes. Maintenant ça progresse vers une certaine égalité, les garçons ont compris également l'intérêt de porter de jolis sous-vêtements. Mais c'est assez récent comme phénomène.

Les garçons sont plus à l'aise dans leur nudité. Ça ne les dérange pas. Les petites filles sont très tôt sexualisées. Je ne crois pas que ce soit génétique, mais plutôt culturel. Très tôt, la jeune fille est consciente de sa séduction. Les petites filles sont très influencées par les chanteuses pop. Les petites filles sont hyper sexualisées, elles grandissent dans la séduction. Aujourd'hui, les garçons rattrapent ça. On va de plus en plus vers une féminisation du corps masculin, particulièrement dans les vêtements, et dans l'attitude.

CLAIRE VIONNET : Dans l'ordinaire, il y a une association entre nudité et sexualité, ce que les chorégraphes contemporains semblent dissocier.

Daniel Léveillé : Dans toutes les pièces que j'ai faites avec le nu, on n'est jamais vraiment dans la sexualité. La sexualité n'est que l'une des nombreuses autres composantes de l'être humain. Elle n'est pas niée, pas plus que soulignée. Ce ne sont pas des pièces antisexuelles, mais on n'est pas explicitement dans la sexualité. Souvent, des parents me demandent s'ils peuvent venir avec leurs enfants de quatre 
ou cinq ans. Comme il n'y a aucune ambiguïté dans ces pièces, même un jeune enfant peut y être exposé. Je ne mets pas d'emphase sur la sexualité. Je mets l'emphase sur la netteté des intentions : est-ce que nous sommes dans un rapport de sexualité ? Non, mais de séduction certainement, on ne peut pas l'éviter. Il y a toujours l'ombre de la possibilité d'un rapport de sexualité dans un contact entre deux personnes: un couple d'homosexuels va sûrement y lire un rapport de sexualité alors qu'une mère $y$ verra plutôt une relation entre deux frères ou entre deux amis. Un hétéro va y lire sa relation avec son meilleur ami et cette relation n'est pas sexuelle. La nudité peut ne pas être sexuelle et c'est le cas dans mon travail : deux garçons ensemble, c'est un lien homosexué mais pas nécessairement sexuel. Il peut exister un lien très fort entre deux hommes qui ne soit pas sexuel. Idem pour un duo entre deux femmes.

CLAIRE VIONNET : Et qu'en est-il de l'association à l'érotisme?

Daniel Léveillé : Pour qu'il y ait érotisme, il faut être habillé. Dès qu'on est nu, on n'est plus dans l'érotisme. Est-ce que mes pièces érotisent? Je crois que non. Habillé minimalement, là on serait dans une sorte d'érotisme. Mais avec la nudité, l'érotisme est entièrement évacué. Ou alors, l'érotisme est présent parmi toutes les autres composantes des danseurs sur scène. Il n'y a pas de geste au premier degré qui propose quoi que ce soit de cet ordre-là. Si je relève ma jupe devant les spectateurs ou que je me dévêts une épaule, là ce serait très clair. Si la chorégraphie le demande, l'érotisme est très facilement utilisable. Et la proposition serait claire.

Tant qu'il reste une petite culotte à enlever, un soutien-gorge ou même un bijou, on reste dans un jeu de séduction. Par contre, le jeu de séduction n'est plus possible une fois nu. Quand tout est enlevé, on ne peut plus être dans la séduction. Comment faire ce geste-là (se dévêtir) sans titiller l'audience? Comment enlever un jeans serré, comment enlever un sous-vêtement, un slip assez ajusté et le glisser entre les deux jambes? Comment veux-tu faire ça sans être dans le sexuel? Impossible! Dans la sexualité, on arrive toujours à l'étape pendant laquelle on se dévêt. Ça ne se passe jamais dans mes pièces. Beaucoup de chorégraphes le font sur le côté, plutôt qu'au centre de la scène, sous la lumière. Inévitablement, c'est une proposition savoureuse sinon « titilleuse ».

Pour moi, l'érotisme est lié au voilé et à la suggestion. Ne laisser qu'entrapercevoir. Ou alors dans l'action de se dévêtir. La nudité en soi n'est ni érotique, ni sexuelle. Je ne focalise pas là-dessus, je ne rends pas ces corps sexuels. Du début à la fin dans mes pièces, les danseurs sont nus. Ils ne passent jamais par l'étape de se dévêtir. Baudrillard a écrit « la séduction est courbe ", ce qui est très juste. De là à penser que la nudité est droite et franche...

\section{Entre accueil et réticence : politiques de réception}

CLAIRE VIONNET : Comment tes pièces ont-elles été reçues au Québec, une région plutôt réfractaire à la nudité, me semble-t-il ?

Daniel Léveillé : À Montréal, elles ont été très bien reçues. Au début, en 2001, j'étais un peu réticent. Je voyais bien qu'il y avait des voyeurs dans la salle. C'est toujours les deux premières rangées et ils étaient facilement identifiables: des hommes d'un certain âge. Ça me dérangeait un peu, jusqu'au jour où je me suis dit: "pourquoi pas? » Ces hommes-là en ont probablement besoin; ils viennent d'une génération où 
leur sexualité a été probablement excessivement frustrée pour toutes sortes de raisons. Ils viennent voir des corps nus. Cela correspond certainement à un besoin. Ils réalisent bien qu'ils ne sont pas dans un bar de danseuses ou de danseurs nus. Des connaissances et des ami-e's très âgée's viennent également voir mes pièces. Pour eux·elles, c'est comme une thérapie. C'est du pur bonheur pour ces gens-là. Ça les libère. Ces gens-là peuvent se dire : « c'est moi, j'avais ce corps-là ». Quand on regarde la danse, on la vit, on devient ce protagoniste-là, qui danse sur scène. Ces vieux corps deviennent, le temps du spectacle, ces beaux jeunes corps. Ça devrait être obligatoire dans les centres de personnes âgées, pour les sortir un peu du désastre absolu de leurs vieux corps qui s'écroulent. Je trouve ça très thérapeutique, très sain.

Dans les années 1970, l'utilisation de la nudité sur scène n'était pas du tout la même. Ce n'était pas du tout perçu de la même manière. Le Québec d'alors était encore une société relativement religieuse, même après la révolution sexuelle de la fin des années 1960. C'était encore très religieux ici. Il y avait une autocensure sexuelle, probablement suite aux années où l'Église contrôlait tous les aspects de la sexualité. Même la danse, même le ballet classique y était alors mal vu par l'Église. L'utilisation de la nudité sur scène participait dès lors d'une revendication du corps et d'une libération sexuelle. Le geste était politique, il n'était pas neutre. D'ailleurs, mes pièces de cette époque étaient plus théâtrales que celles d'aujourd'hui. Il y avait le besoin d'inscrire cette contestation dans la société d'alors. C'était plus qu'un changement de générations, ça a été un changement de société que l'histoire a nommé la révolution tranquille. Alors quand en 2001 j'ai utilisé la nudité, avec une pièce entièrement nue du début à la fin pour les quatre danseurs, il n'y avait plus aucune revendication particulière, ni sexuelle, ni politique.

\section{CLAIRE VIONNET : Et comment tes créations sont-elles reçues ailleurs dans le monde?}

Daniel Léveillé : Elles y ont été également très bien reçues. J'ai essentiellement tourné ces pièces-là en Europe, mais pas seulement. J'ai quand même fait Israël et l'Indonésie (Jakarta). Faut le faire, dans un pays musulman! Aucune publicité n'a été faite pour la pièce pour ne pas alerter, et tous les spectateurs potentiels ont été prévenus plutôt discrètement. À Tel Aviv, ça s'est passé différemment. Il y a eu des polémiques dans les journaux, les ultras orthodoxes n'étaient pas très contents d'avoir des « tous nus » chez eux. Sinon, on a tourné presque exclusivement dans des pays européens. S'il y en a vingt-huit, on a dû en faire autour de vingt-quatre. Surtout en France et en Allemagne, mais un peu partout également, du Nord au Sud et de l'Ouest à l'Est.

L'endroit où ça a été le moins évident d'abord, étonnement, c'est la France. Les gens étaient très réticents au début, en 2002. Mais j'ai eu la chance de donner beaucoup d'entrevues, notamment à la radio, à des émissions d'assez bonne cote, et j'ai bénéficié d'une présence médiatique assez exceptionnelle dans des journaux. J'ai également eu beaucoup de discussions avec le public après les spectacles. J'ai réalisé que les Français, il faut qu'ils comprennent. Tant qu'ils ne comprennent pas le pourquoi de ce geste-là, ils rechignent. Beaucoup de spectateurs français sont quand même venus voir mes pièces et puis, ils ont bien vu que le geste était tout sauf gratuit. Depuis, c'est devenu un peu une belle histoire d'amitié entre eux et moi et j'ai pu beaucoup présenter mon travail en France. Il y a deux petits pays où j'ai présenté toutes mes pièces dans les villes d'importance, deux petits pays où je suis vraiment une vedette : la Suisse et le Danemark. Lorsque je vais dans ces deux pays-là, si je ne 
fais pas la une des journaux, je fais assurément la page couverture du cahier Arts et Lettres.

CLAIRE VIONNET : Ton récit détourne les clichés d'un Occident soi-disant émancipé et de pays religieux pudiques. II y a des courants réceptifs et résistants partout, de sorte que les généralisations ne sont pas possibles. Comment peut-on expliquer cette réception plus favorable dans certains endroits que d'autres?

Daniel Léveillé : On n'y échappera jamais, il faut que les diffuseurs soient intéressés par le travail. Ce sont eux qui choisissent les pièces. J'ose penser que les diffuseurs aiment mon travail, et qu'ils m'apprécient moi aussi en tant qu'artiste. Ce sont eux d'abord qui savent quoi faire et comment faire pour qu'une œuvre soit bien reçue. Il y a toujours le bon moment et le bon lieu. En Suisse, mon travail a un véritable impact. À Genève, on fait toujours quatre ou cinq représentations devant des salles combles. Qu'est-ce qui explique cela? Je ne saurais dire, mais la personne qui nous reçoit y est assurément pour quelque chose.

En 2003-2004, on a dansé à Rome, et j'ai alors pensé que la pièce s'inscrivait dans cette ville à cause des sculptures extérieures présentes partout. En France, dans les jardins publics, il y a surtout des sculptures de nus du XIX siècle. À Rome, c'est plus ancien, plus classique, même plusieurs sculptures antiques monumentales. Dans d'autres villes, c'est surtout la sculpture monumentale de nus art déco qui prédomine. L'esthétique de ces sculptures extérieures, véritable histoire de la ville dans la pierre, peut être assez différente d'une ville à l'autre. La présence de ces corps nus dans la ville m'amène à penser que la perception de corps nus sur scène, par les spectateurs, peut en être influencée; ces spectateurs, qui vivent dans ces villes-là, côtoient quotidiennement ces sculptures-là et peut-être que ça change un peu leur perception de la nudité sur scène.

Aujourd'hui, il y a beaucoup de nudité sur les scènes de danse contemporaine. La quantité de jeunes chorégraphes, garçons et filles, qui vont faire des spectacles solos et des spectacles nus, c'est assez étonnant. Ça s'est vraiment multiplié.

CLAIRE VIONNET : Pourquoi penses-tu qu'il y a une plus grande acceptation de la nudité aujourd'hui ?

Daniel Léveillé : Il faut bien avouer que l'on vit une période extraordinaire - en Occident du moins - de libération des corps. Ce n'est pas qu'une libération sexuelle, parce qu'il reste encore beaucoup de chemin à faire à ce sujet et en particulier en termes d'égalité entre les hommes et les femmes. Mais une libération dans le sens d'une acceptation des corps. Tout le mouvement transgenre aurait été impossible il y a à peine trente - quarante ans. La révolution féministe a mené à l'acceptation de l'homosexualité, des transgenres, et de tout ce qui est autre que le «mâle alpha dominant ». C'est fabuleux! Et ce n'est pas terminé cette histoire-là. Est-ce qu'il y aura des reculs? Fort probablement. L'histoire de l'humanité a toujours été cyclique, mais c'est quand même fantastique ce qu'on peut vivre aujourd'hui dans nos sociétés. Pour les artistes, c'est une période assez riche de potentiels. Et il est possible de ne pas passer l'essentiel de son temps à dénoncer. Même s'il y a toujours un mouvement de l'art qui est un mouvement très politique et très social et que ce mouvement est encore très pertinent, ce n'est pas ma tasse de thé ; mon intérêt premier est l'art pour l'art.

Je suis très lié au mouvement moderne qui travaille les paramètres premiers, dont la forme. Mon écriture se simplifie de plus en plus. Si j'étais un artiste visuel, j'en serais 
à ma période monochrome. Enlever tout le superflu et ne laisser que le minimum. C'est ça qui est beau. La création, c'est comme la vie. À un moment donné, la vie s'arrête. Parce qu'on est vieux, parce qu'on meurt. La création, c'est comme ça. On se demande toujours ce qui arrivera quand on ne sera plus inspiré. Mais ça n'arrivera pas. La création, c'est une pulsion première comme la pulsion sexuelle, comme la pulsion de manger et de boire pour survivre, c'est là. Il y a des périodes plus creuses et c'est normal, un peu comme dans la vie. Il y a des moments et des cycles qui diffèrent, mais la création ne meure pas.

\section{BIBLIOGRAPHIE}

ANDRIEU Bernard, « Quelle épistémologie du corps ? », Corps, n 1, vol. 2006, 2006, pp. 13-21.

ANDRIEU Bernard, BÖETSCH Gilles, Corps du monde. Atlas des cultures corporelles, Paris, Collin, 2013.

BERNARD Michel, De la création chorégraphique, Pantin, Centre National de la Danse, 2001.

BÖETSCH Gilles, ANDRIEU Bernard, LE BRETON David, POMARÈDE Nadine, VIGARELLO Georges, La Peau. Enjeu de société, Paris, CNRS, 2008.

BOISSEAU Rosia, CROQUET Nadia, Deuxième peau, habiller la danse, Galerie d'Art du Conseil Général des Bouches-du-Rhône (catalogue d'exposition), Aix-en-Provence, Actes Sud, 2005.

DORAIS Michel, Petit traité de l'érotisme, Montréal, VLB éditeur, 2010.

DÜRR Hans Peter, Nudité et pudeur. Le mythe du processus de civilisation, Paris, Maison des Sciences de l'Homme, 1998.

DUVIGNAUD Jean, KHAZNADAR Chérif, Le Corps tabou. Internationale de l'imaginaire, vol. 8, Paris, Maison des cultures du monde, 1998.

FABBRI Véronique, Penser la danse contemporaine, vol. 44, Paris, Presses Universitaires de France, 2004.

HUESCA Roland, « Danser nu : usage du corps et rhétorique postmoderne », Symposium : Revue Canadienne de Philosophie Continentale, $\mathrm{n}^{\circ} 2$, vol. 10, 2006, pp. 569-586.

HUESCA Roland, « Nudités, les "utopiques" du corps et de la peau ", Agôn : Utopies de la scène/ scènes de l'utopie, $\mathrm{n}^{\circ}$ 3, 2010, [en ligne], https://journals.openedition.org/agon/1474, page consultée le 6 octobre 2020.

HUESCA Roland, La nudité (3/5) : Danser nu, jouer nu. Usage de la nudité dans le spectacle vivant, Émission Pas la peine de crier, France Culture, 10 juillet 2013, [en ligne], http://

www.franceculture.fr/emission-pas-la-peine-de-crier-la-nudite-35-danser-nu-jouer-nu-usagede-la-nudite-dans-le-spectacle-, page consultée le 20 avril 2020.

HUESCA Roland, « Nudity and figures of transgression at the end of the 20th century ", Dancehouse diary, $\mathrm{n}^{\circ}$ 6, 2014, pp. 4-6.

HUESCA Roland, La Danse des orifices. Étude sur la nudité, Paris, Nouvelles Éditions Jean Michel Place, 2015. 
HUSEMANN Pirkko, Ceci est de la danse. Choreographien von Meg Stuart, Xavier Le Roy und Jérôme Bel, Norderstedt, Books on Demand GmbH, 2002.

KENNETH Clark, The Nude: A Study in Ideal Form [1956], New Jersey, Princeton University Press, 1990.

LE BRETON David, La chair à vif, Paris, Métailié, 1993.

LE BRETON David, Anthropologie du corps et modernité [1990], Paris, Presses Universitaires de France, 2005.

LOUPPE Laurence, Poétique de la danse contemporaine, Bruxelles, Contredanse, 1997.

PASTORI Jean-Pierre, À corps perdu. La danse nue au XX siècle, Lausanne, Pierre-Marcel Favre, 1983.

PERRIN Julie, « Le nu féminin en mouvement », in BIET Christian, ROQUES Sylvie (dir.),

Communications. Performance. Le corps exposé, vol. 92, Paris, Le Seuil, 2003, pp. 173-182.

PSAROLIS Alexia, « Nudités plurielles. Entretien avec Roland Huesca », Nouvelles de danse, 2015, [en ligne], http://revues.be/nouvelles-de-danse/118-automne-2015/249-dossier-nuditesplurielles-entretien-avec-roland-huesca, page consultée le 6 octobre 2020.

RANCIÈRE Jacques, Le spectateur émancipé, Paris, La fabrique Éditions, 2008.

TURNER Terence S., " The Social Skin ", in LOCK Margaret, FARQUHAR Judith (dir.), Beyond the Body Proper: Reading the Anthropology of Material Life (body, commodity, text), Durham/London, Duke University Press, 2007, pp. 83-103.

\section{NOTES}

1. TURNER Terence S., " The Social Skin », in LOCK Margaret, FARQUHAR Judith (dir.), Beyond the Body Proper: Reading the Anthropology of Material Life (body, commodity, text), Durham/London, Duke University Press, 2007, pp. 83-84.

PSAROLIS Alexia, « Nudités plurielles. Entretien avec Roland Huesca ", Nouvelles de danse, 2015, [en ligne], http://revues.be/nouvelles-de-danse/118-automne-2015/249-dossier-nuditesplurielles-entretien-avec-roland-huesca, page consultée le 6 octobre 2020.

2. HUESCA Roland, La nudité (3/5): Danser nu, jouer nu. Usage de la nudité dans le spectacle vivant, Émission Pas la peine de crier, France Culture, 10 juillet 2013, [en ligne], http:// www.franceculture.fr/emission-pas-la-peine-de-crier-la-nudite-35-danser-nu-jouer-nu-usagede-la-nudite-dans-le-spectacle-, page consultée le 20 avril 2020.

3. KENNETH Clark, The Nude: A Study in Ideal Form [1956], New Jersey, Princeton University Press, 1990, p.3.

\section{RÉSUMÉS}

Cette conversation avec le chorégraphe montréalais Daniel Léveillé met en exergue les enjeux de la nudité en danse contemporaine. Si le corps nu est usuel sur les plateaux scéniques, il pose néanmoins des défis à la création. La discussion en interroge les usages, significations, 
représentations, l'association aux notions d'intimité et de sexualité, de gêne et de pudeur. Le phénomène du nu est questionné depuis le point de vue chorégraphique, donnant voix aux intentions du chorégraphe. Les politiques de réception sont également évoquées, soulignant la diversité des modes de réception à l'échelle internationale. Enfin, l'exhibition du nu est pensée dans son enchevêtrement avec d'autres médias, tels que la lumière, le geste et l'espace.

This conversation with Montreal's choreographer Daniel Léveillé shows controversies about nudity in contemporary dance. Although naked bodies are usual on stage, they nevertheless challenge choreographic creation. The discussion investigates the uses, significations and representations of the naked body, its association with notions of intimacy and sexuality, shame and pudor. The phenomenon is tackled from a choreographic perspective, giving a voice to the choreographer's intentions. Politics of reception are portrayed, underlying the diversity of modes of reception on the international level. Finally, the exhibition of naked dancing bodies is conceived in articulation with other medias such as light, gestures and space.

\section{INDEX}

Keywords : Intimacy, nudity, production process, Quebec, sexuality

Mots-clés : Intimité, nudité, processus de création, Québec, sexualité

\section{AUTEURS}

\section{DANIEL LÉVEILLÉ}

Formé au sein du Groupe Nouvelle Aire, Daniel Léveillé travaille longtemps comme chorégraphe indépendant avant de fonder Daniel Léveillé Danse en 1991. Tandis qu'il signe des œuvres pour diverses compagnies de danse et de théâtre, il intègre le département de danse de l'Université du Québec à Montréal et y occupe un poste de professeur dans le champ de la création et de l'interprétation en danse (1988 - 2012). En 2001, Amour, acide et noix (2001) établit sa réputation sur la scène internationale où il y présentera par la suite La pudeur des icebergs (2004) et Crépuscule des océans (2007). Il développe alors une écriture faite de répétitions et de phrases courtes, imagine des partitions chorégraphiques à la limite de l'impossible qui contribuent à révéler la beauté de l'être dans toutes ses imperfections et il choisit la nudité comme seul costume possible. Avec Solitudes solo (2012), il initie un nouveau cycle de création où l'on voit poindre une gestuelle plus fluide, le retour d'un costume autre que la nudité. En 2018, Daniel Léveillé quitte ses fonctions de direction artistique de la compagnie mais demeure actif à titre de chorégraphe.

\section{CLAIRE VIONNET}

Claire Vionnet est anthropologue et chercheuse en danse au Fonds National Suisse de la recherche scientifique. Depuis 2013, elle s'intéresse aux processus de création chorégraphique à partir d'une ethnographie auprès de compagnies de danse contemporaine en Suisse. Sa recherche actuelle, en collaboration avec la Faculté des Beaux-Arts de l'Université Concordia de Montréal et le Département danse de Paris 8, porte sur le phénomène de l'intime en danse contemporaine et en contact improvisation. Ses publications questionnent les identités artistiques (2015), les politiques de réception (2017), la sensorialité (2018), la collaboration entre art et anthropologie (2018), l'intimité (2019), le geste $(2018,2020)$, ainsi que l'enchevêtrement entre danse et rituel (2020). 